\title{
TRACTION AND WEAR MECHANISMS DURING ROLL-SLIP CONTACT
}

\author{
J. De Pauw ${ }^{1}$, J. Van Wittenberghe ${ }^{1}$, P. De Baets ${ }^{1}$ \\ ${ }^{1}$ Ghent University, Laboratory Soete, Belgium
}

\begin{abstract}
In the transportation industry every vehicle that makes contact with the road and propels itself, is subjected to roll-slip in the wheel-road interface. Therefore a good understanding of this phenomenon is important. This article describes roll-slip contacts in general, with extra attention for the wheel-rail contact which is commonly used. Firstly the contact area and the pressure distribution that occur when a normal load is applied between two bodies is discussed. On this base, the traction mechanisms for static and rolling configurations are shown. Lastly, wear mechanisms and two different wear maps are shown which are very useful and commonly used in wear mapping of roll-slip contacts. A good agreement with field measurements can be obtained.
\end{abstract}

Keywords Roll-slip contact, wear, traction, friction

\section{INTRODUCTION}

The contact mechanics in rolling and roll-slip problems have been investigated for a long time, in particular in transport engineering, where the oldest frequently cited paper dates from 1926 [1]. In this sector traction is needed for the propulsion of trains, cars and other vehicles. A high traction coefficient is required for fast acceleration and braking. To optimize this traction, a theoretical description is needed. This optimization is not only beneficial for faster accelerating and braking, a higher traction coefficient can also lead to vehicles that are able to drive on steeper slopes.

Roll-slip contact also occurs in gears, bearings and transmission belts, where the traction coefficient is a disadvantage and a source of energy loss.

In a gear the entire interacting tooth flanks roll and slip simultaneously over each other except at the pitch diameter, where the contact is only obtained by pure rolling. Traction between the tooth flanks is undesirable because it causes an opposing torque, generates heat, and encourages rolling contact fatigue which can lead to surface fatigue [2].

Research in roll-slip problems results in a better understanding of the mechanisms, and lead to more accurate theories. These theories can be used to develop higher traction coefficients and a better wear control, which is beneficial for traction applications.

In this article we will focus on roll-slip problems in general, with special attention to wheel-rail contact which are often used in the railway industry.

The contact area and corresponding pressure distribution will be discussed for simple contact geometries and more complex wheel-rail contact geometries.

Consecutive, traction modeling in the contact area is discussed. This modeling is necessary for traction applications.

Finally, we highlight wear mechanisms and wear regimes in roll-slip contacts, since they affect the previously discussed points, and since wear is an important economical factor. Again the wheel-rail problem will be used as example.

\section{CONTACT MODELING}

The contact between two surfaces gives rise to an area of contact, and a pressure distribution over this contact area. If the surfaces have a simple geometry, the contact theory of Hertz (1881) can be used for calculating the contact area and the pressure distribution. For contacting surfaces that have a more complex shape, there is in most cases no closed form analytical solution. Boundary- and finite element programs $[3,4]$ can calculate the contact area and the associated pressure distribution. 


\subsection{Hertz contact}

As previously stated the Hertz contact theory can only be used if the contacting bodies have a simple geometry. A geometry is simple if the curvature of the body is constant, and has at most two different curvatures in perpendicular direction, like a smooth sphere, smooth cylinders or flat plane. The Hertz contact theory can be used for all combinations of the three previous states bodies, accept the plane-plane contact.

Beside the geometry condition, two other conditions should by fulfilled. The deformation has to be fully elastic and the surfaces are supposed to be frictionless.

If two bodies touch each other in the absence of a normal load, there will be a point or line contact, depending on the contacting surfaces. An initial point contact will grow with increasing load to a circular or an elliptical contact area, while a line contact will develop to an elongated elliptic contact.

In the case of a sphere with a radius $R_{1}$ loaded against a flat plane with a force $F$, the radius $a$ of the contact area will be:

$$
a=\sqrt[3]{\frac{3}{2} \frac{F \cdot R_{1}}{E^{\prime}}}
$$

Where $E^{\prime}$ is the effective modulus of elasticity, defined by:

$$
\frac{1}{E^{\prime}}=\frac{1-v_{1}^{2}}{2 E_{1}}+\frac{1-v_{2}^{2}}{2 E_{2}}
$$

The pressure distribution is parabolic over the contact area, with the maximum pressure $p_{\max }$ occurring in the centre of the contact area.

$$
p_{\max }=\frac{3 \cdot P}{2 \cdot \pi \cdot a^{2}}
$$

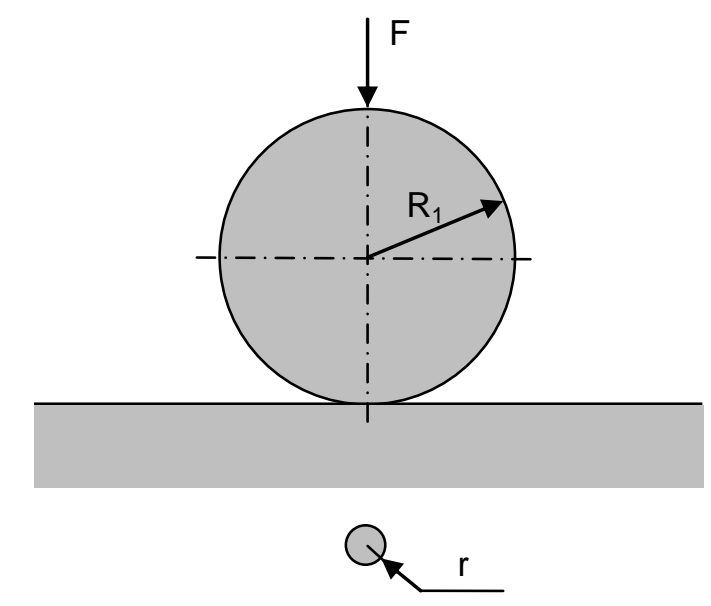

Figure 1. Contact area in a sphere-plane contact

The contact theory of Hertz assumes smooth surfaces, so no surface roughness is taken into account. However, every engineering surface has a certain surface roughness. The simplest model to take surface roughness into account is that of Archard [5], where the surface of the sphere with radius $R_{1}$ is covered with an evenly distribution of identical spherical protuberances of radius $R_{2}$, where $R_{1}>>R_{2}$. A mathematical solution for the contact area is available.

More recent experimental research about the influence of surface roughness specific in roll-slip contact is given by L. Xiao et al. [6]. Where it is found that rough surfaces have a higher friction coefficient than smooth surfaces, and the friction coefficient is function of the slip $S$. 


\section{$2.2 \quad$ Non Hertz contact}

If the interacting surfaces have a more complex shape, boundary - and finite element programs can be used to calculate the contact area and the corresponding pressure distribution. In contrast to the Hertz contact model, it is not necessary that the behavior is fully elastic; rough surfaces and strain hardening can be included in the model.

Roll-slip contact between complex shapes can be found in particular in the railway industry, where steel wheels running on steel rails provide the necessary traction for propulsion. The contact area and the pressure distribution can by calculated by general finite element programs like Abaqus ${ }^{\circledR}$ or Ansys ${ }^{\circledR}$. Wheel/rail contact simulations can also be performed by dedicated programs, each with their specific algorithm, like Contact [7], Fastsim [7] or Non-Hertz [8].

The first two algorithms are given by Kalker, who uses a half-space assumption that is not restricted to elliptic contact. The Contact program divides the contact area in small rectangular elements in which the traction is constant, the adjacent elements interact with each other using an influence function approach.

The weakness of this program is the numerical integration that is required, so online calculations for transient vehicle simulations are impossible. The Fastsim algorithm uses the Kalker's simplified theory, in which a constant stress gradient is assumed until the limiting traction force is reached. The traction bound is defined as the normal force multiplied by the coefficient of friction.

Figure 2 shows a $\mathbf{S} 1002$ wheel profile and a UIC60 rail profile. Depending on their relative position to each other, the position and shape of the contact area vary, as clearly shown in Figure 3. Also the pressure distribution will be affected.

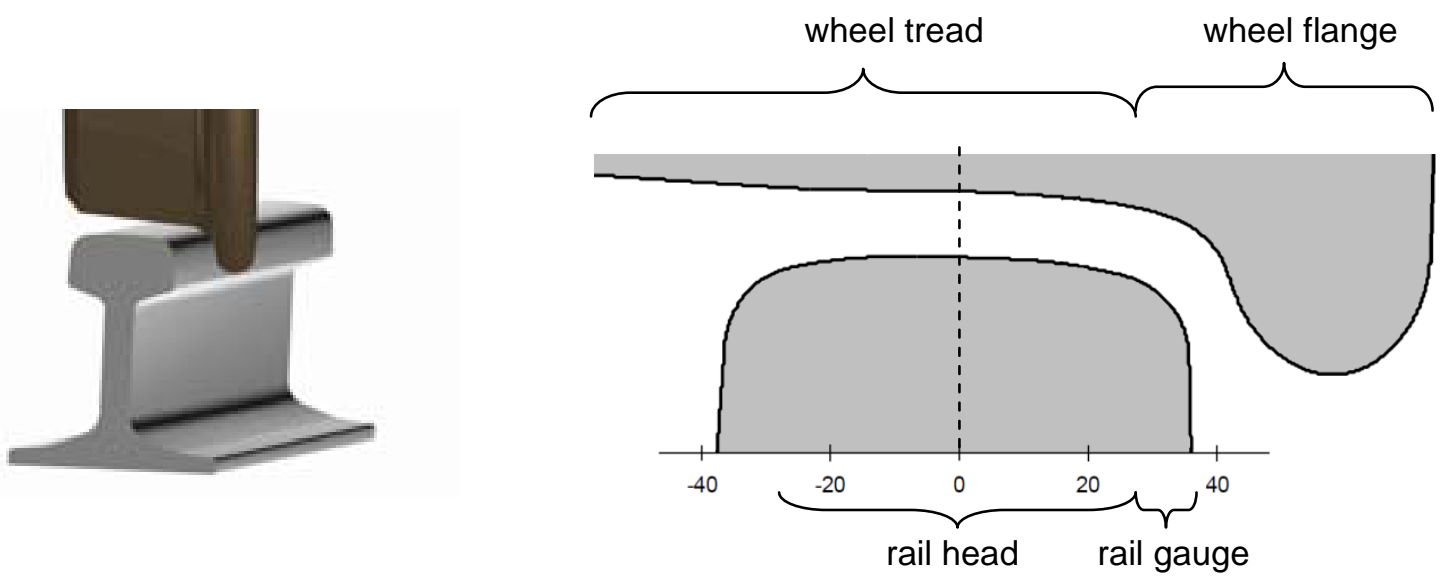

Figure 2: left: general view, right: surface detail with wheel profile S1002 and rail profile UIC60

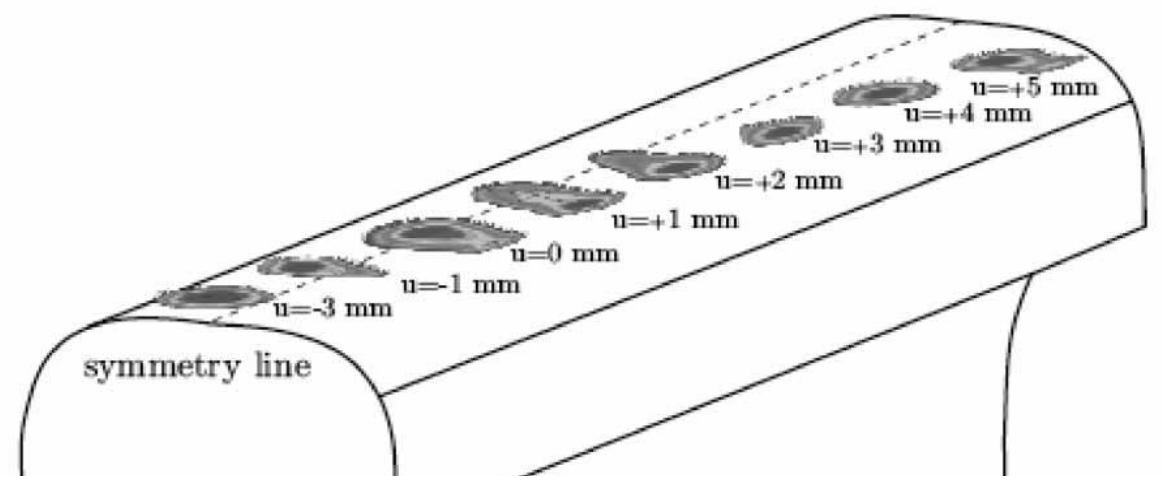

Figure 3. Comparison of the position and the shape of the contact area, depending on the variation of the lateral shift $u$ (positive towards the gauge face) [9]

If the lateral displacement $u$ increases extraordinary, there will be contact between the wheel flange and the rail gauge. This additional contact disturbs the common contact area between the rail head and the wheel tread, leading to a contact area that is characterized by two pressure peaks, as shown in Figure 4. 


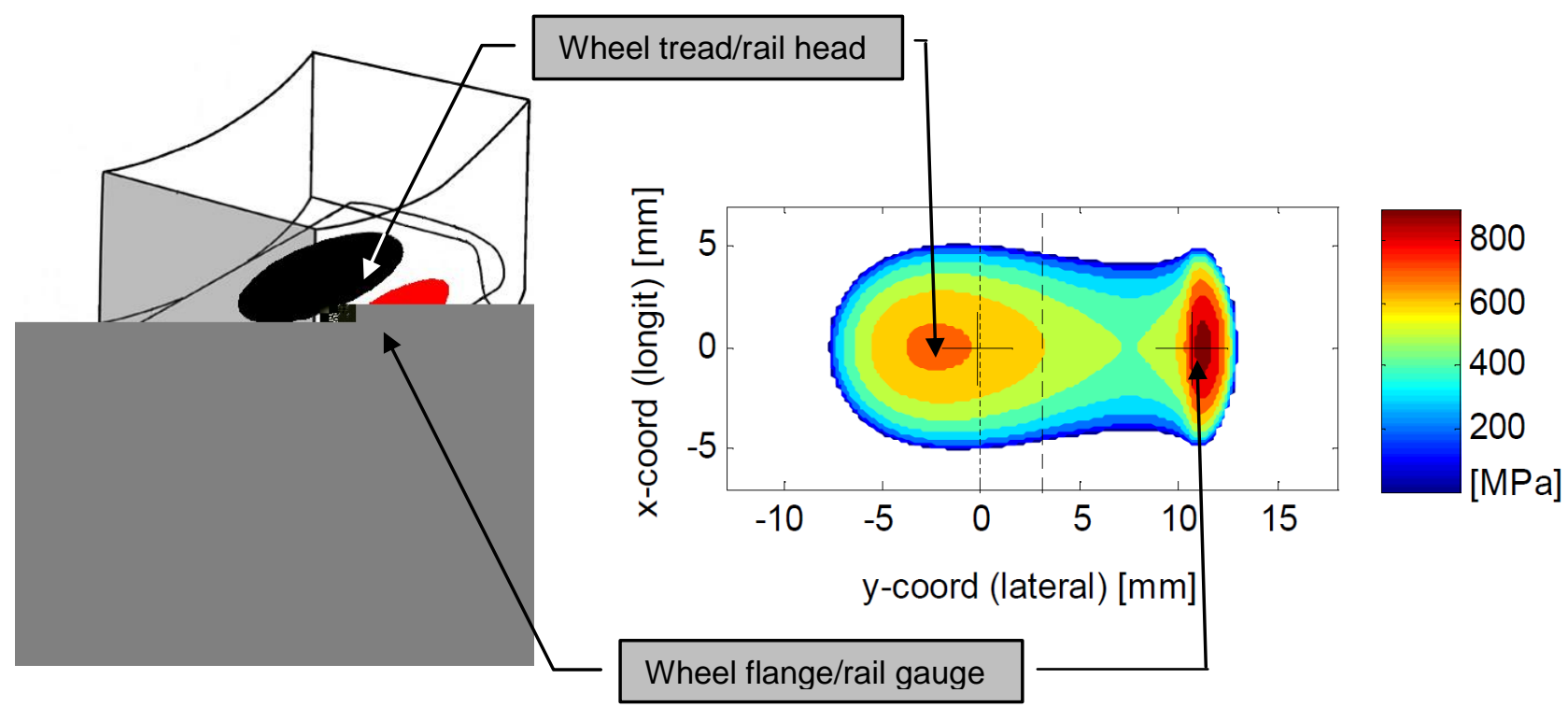

Figure 4: Wheel flange/rail gauge contact, left: overview, right: pressure distribution

\section{TRACTION MODELLING}

Traction is required between two bodies if propulsion or braking takes place. This phenomenon has to be well understood and controlled for accurate function of trams or trains.

When a vehicle accelerates, no excessive macroscopic slip may take place, otherwise the vehicle will hardly depart, and significant wear will occur on the wheels and the rails.

In the case of braking, too much macroscopic slip is also unacceptable because of the high wear rate. A slip ratio of $100 \%$ is totally undesirable for the reason that a flat spot will occur on the wheel, this flat spot affects the roundness of the wheel which leads to vibrations in the rail vehicle, thus decreasing ride comfort.

Traction is often considered as a black box, where only the experimental relation between the traction coefficient and the slip is known (Figure 5). The slip $S$ is defined as the ratio between the sliding velocity and the mean velocity.

$$
S=2 \cdot \frac{v_{1}-v_{2}}{v_{1}+v_{2}}
$$
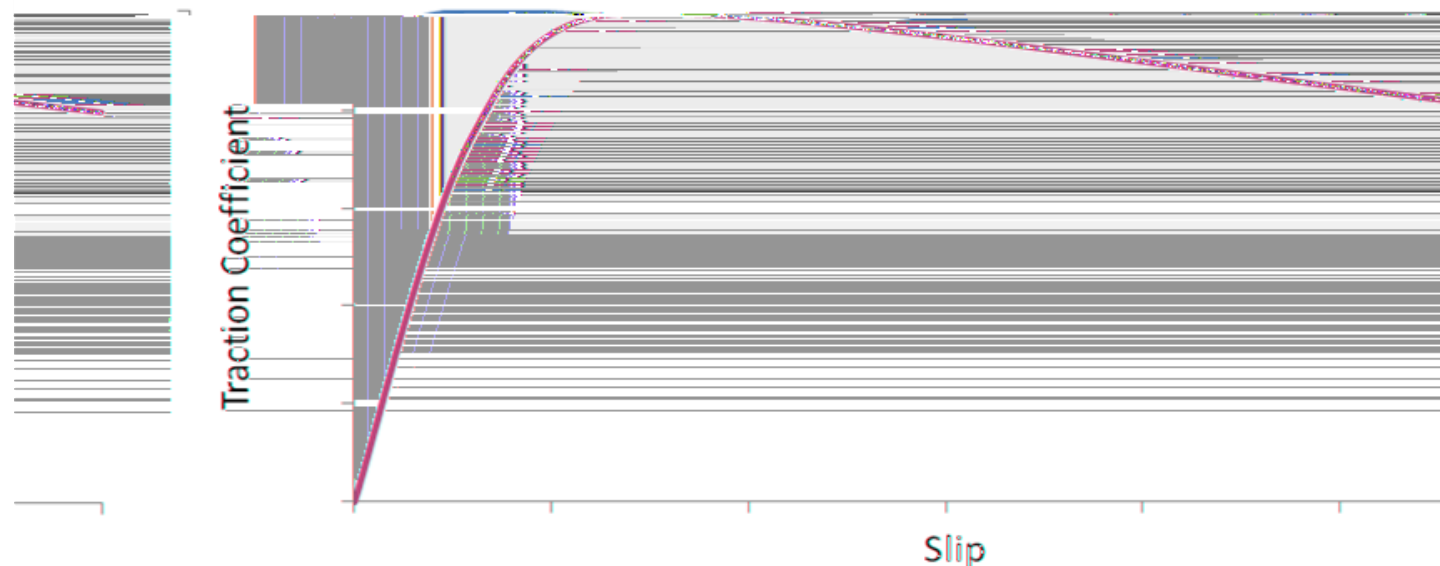

Figure 5: General curve of traction coefficient in function of slip

Pure rolling takes place in the contact area when the surface velocity of the two bodies is equal. In the case of two equal cylinders in contact, they must have the same rotational speed. If the circumference velocity are unequal, the rolling motion is accomplished with sliding.

If two contacting bodies experience a tangential force $Q$, a shear stress will arise in the contact area. No macroscopic sliding takes place if the tangential force is smaller than the limiting friction force $\mu F$. From the 
microscopic point of view, a shear stress $q$ occurs in the contact area. The distribution of the shear stress is not predictable in advance and has to be found by trial and error, until all the specific boundary conditions are fulfilled.

The effect of a tangential force in a static Hertz contact (Figure 1) has been studied by Mindlin [10]. If no relative sliding between the two surfaces takes place, a shear stress $q$ is proposed as shown in Figure 6 . At the edge of the contact the shear stress becomes infinite, which is physically impossible. Mindlin supposed that local slip will occur where the shear stress is bigger than the limiting friction stress $\mu p$. The contact area is divided in two sections, a stick zone where no relative motion between the surfaces takes place, and a slip zone, where the shear stress is the limiting friction stress.

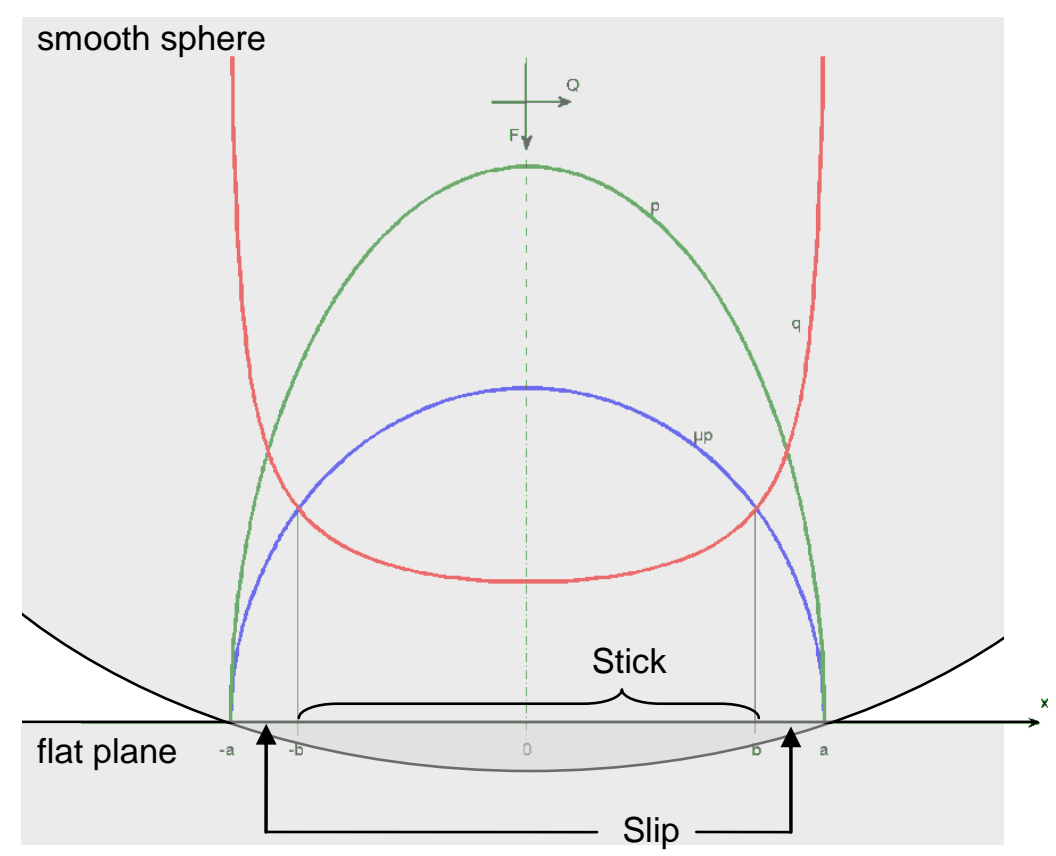

Figure 6: pressure (p) and shear (q) distribution in a static contact area

In pure rolling or roll-slip contacts, the contact area is divided in a stick (pure rolling) and slip region [11]. However the boundary conditions differ from those in the static case, as previously described. In the stick area, no slip may occur (5) and the shear stress must be lower than the limiting friction stress (6)

$$
\begin{aligned}
& S=0 \\
& |q(x, y)|<\mu \cdot p(x, y)
\end{aligned}
$$

In the slip area the shear stress equals the limiting friction stress (7), and the direction of slip must oppose the tangential force (8)

$$
\begin{aligned}
& |q(x, y)|=\mu \cdot p(x, y) \\
& \frac{q(x, y)}{|q(x, y)|}=-\frac{S(x, y)}{|S(x, y)|}
\end{aligned}
$$

Any configuration of stick and slip zone that respects the four boundary conditions is a solution of this problem. For tractive rolling of elastic cylinders which have an elongated elliptical contact area, Carter [1] presented a shear stress distribution $q$ with associated stick and slip area as shown in Figure 7.

If the slip $S$ increases a little, the slip area will be enlarged which leads to a higher amount of shear stress in the contact area. The integral of this shear stress over the contact are is the tractive force, which also enlarges with increasing slip. This relation can be seen on an experimental traction curve (Figure 5) as the rising slope of the curve. 
For pure elliptic or circular contact areas a similar solution can be found. The position of the stick area is located at the trailing edge to respect boundary condition (7), the contour of this area is commonly assumed to be 'pear' or lemon' shaped [11].

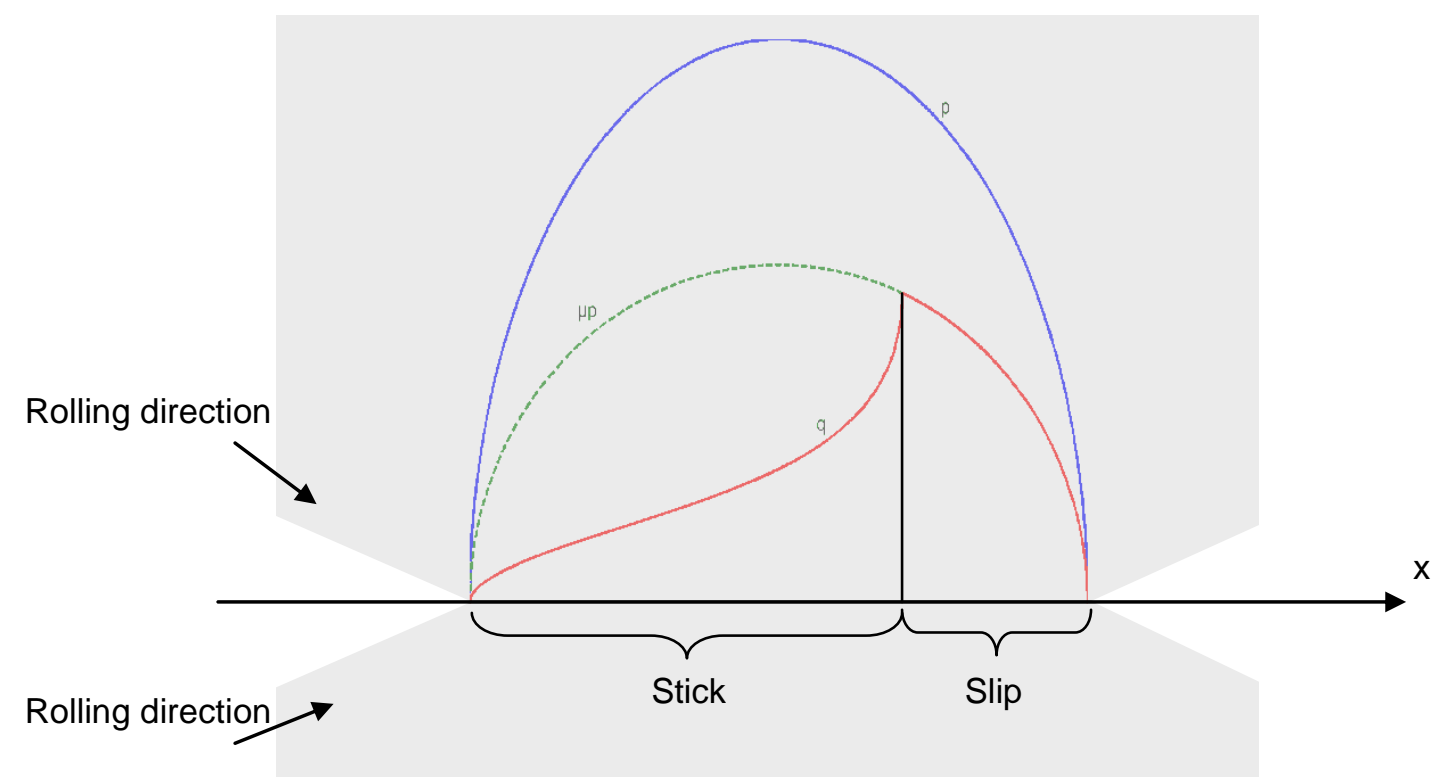

Figure 7: pressure $(p)$ and shear $(q)$ distribution in tractive rolling cylinders

\section{WEAR MODELLING}

In pure sliding problems, the most commonly used wear model is that of Archard [12], where the wear volume $(V)$ is proportional with normal force $(F)$, sliding distance $(s)$ and hardness $(H)$ of the material:

$$
V=K \cdot \frac{F \cdot s}{H}
$$

$K$ is the dimensionless wear coefficient.

In roll-slip contacts two regions can be distinguished. In the stick area no relative motions between the two bodies occur; in the slip area sliding takes place. Equation (9) can be used for modeling wear in roll-slip contacts, where $s$ is the sliding distance in the slip area, or the total macroscopic sliding distance.

In dry or poorly lubricated contact conditions, it is known that the wear coefficient is function of a number of parameters, like normal load, sliding velocity or surface temperature. A sudden change in the wear coefficient indicates a transition in wear mechanisms. The simplest wear classification distinguishes two types of wear: mild and severe wear.

Mild wear results in a surface that is often more smooth than the original surface, with minimal oxide wear debris and plastic deformation. Severe wear is characterized by a rough surface, which is usually rougher than the original surface. In this case a significant amount of plastic deformation, and flake-like wear debris is seen.

The transition between wear regimes is clearly shown in wear maps. In the study area of wear mapping in roll-slip contacts two graphs are frequently used.

In wheel-rail problems the wear mapping of Bolton and Clayton [13] is frequently used, see Figure 8 . The wear rate in $\mu \mathrm{g} / \mathrm{m}_{\text {rolled }} / \mathrm{mm}^{2}$ contact area is plotted against $T S / A$, where $T$ is the limiting traction force (normal force multiplied by the coefficient of friction), $S$ the slip and $A$ the contact area.

A more general view of wear mapping in roll-slip is given by a 3D plot or contour plot that expresses wear volume or wear coefficient in function of normal force and sliding velocity [14-17]. In Figure 9 three boundaries separate the four presented wear regimes: mild, severe, severe-catastrophic transition and catastrophic wear. 


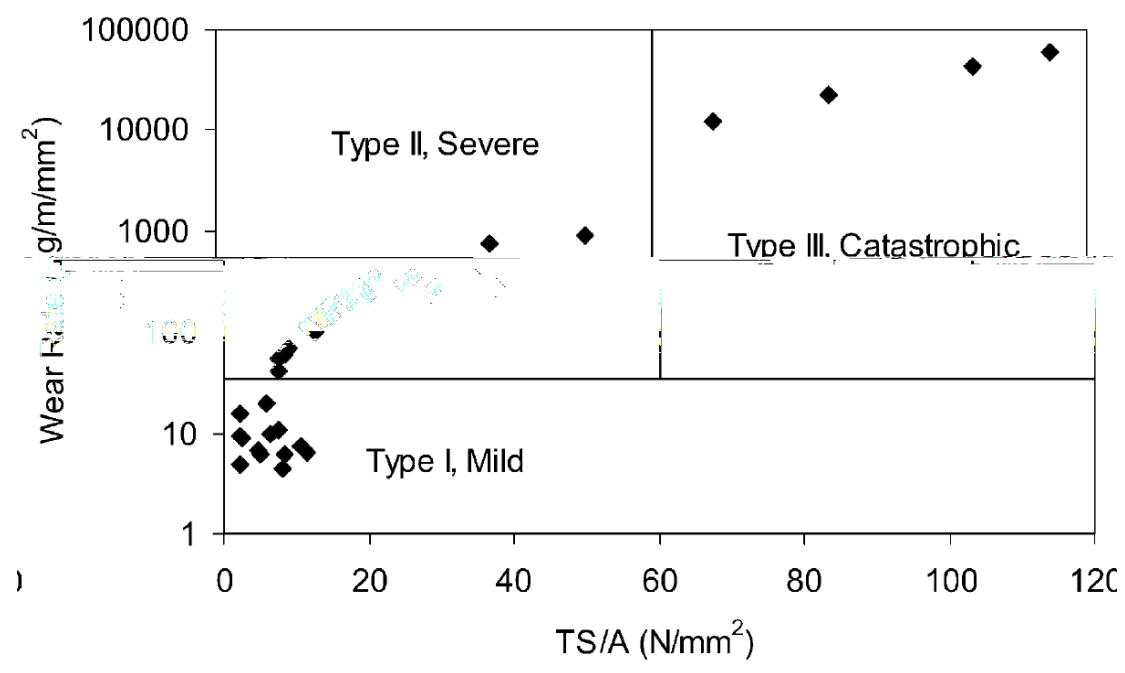

Figure 8: Wear regimes during twin disc testing of BS11 rail material vs. Class D tyre material [13]
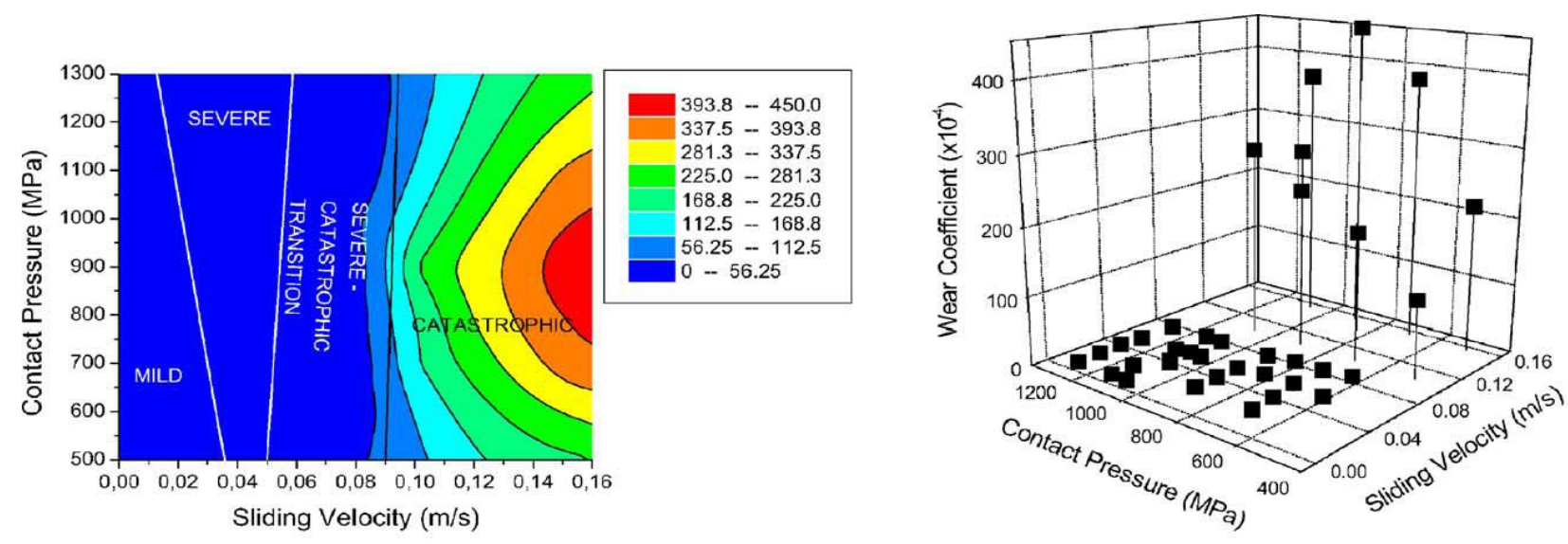

Figure 9: Wear coefficient maps for standard carbon steel. left: contour plot, right: 3D plot [17]

To locate the different wear regimes in wheel-rail contacts, the wear map and the wheel-rail contact conditions map are plotted together on one graph, as shown in Figure 10. This shows that the rail head/wheel tread contact is in the mild to severe wear range while the rail gauge/wheel flange will experience severe to catastrophic wear.

This theory is in good agreement with field measurements of worn rails and wheels [14]. Figure 11 shows the profile of a new rail and a worn rail, these measurements were performed on a straight track. It is clear that the rail gauge/wheel flange contact area is more worn than the rail head/wheel tread contact area. 


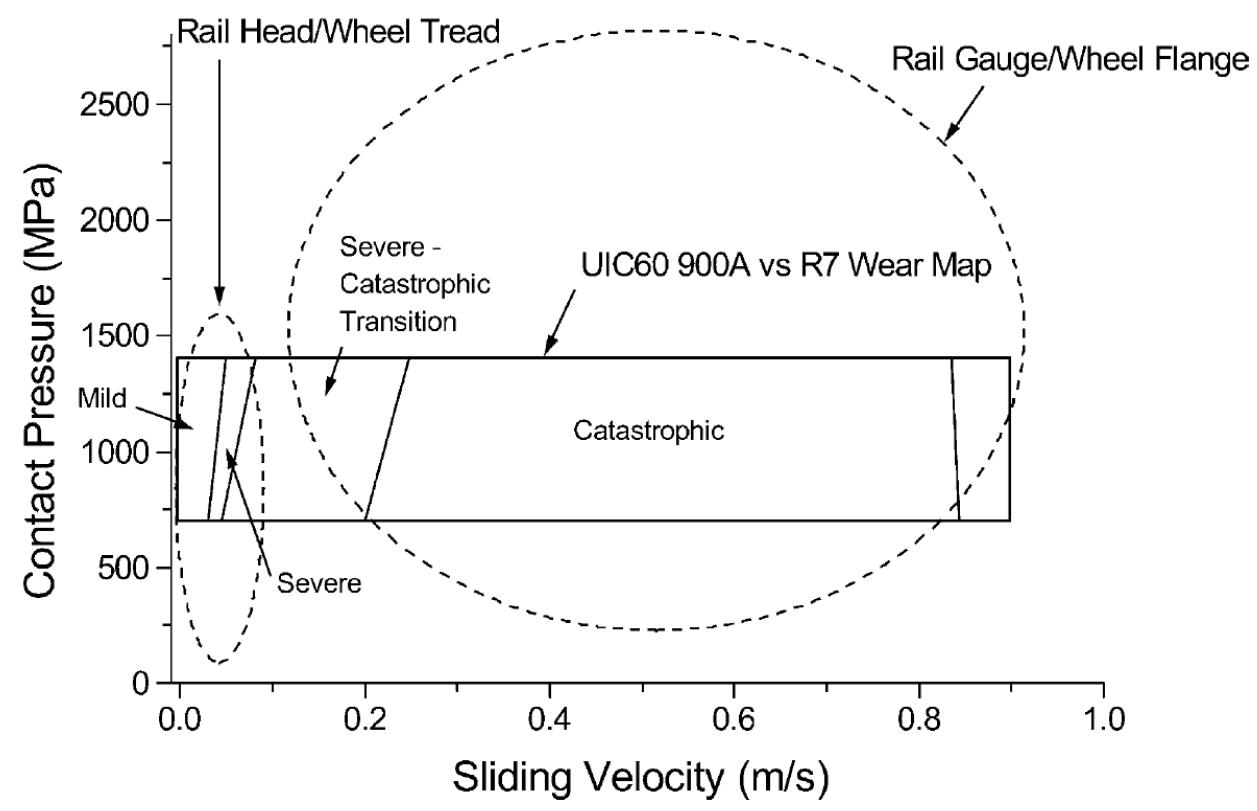

Figure 10: UIC60 900A rail steel wear map over wheel-rail contact conditions [15]

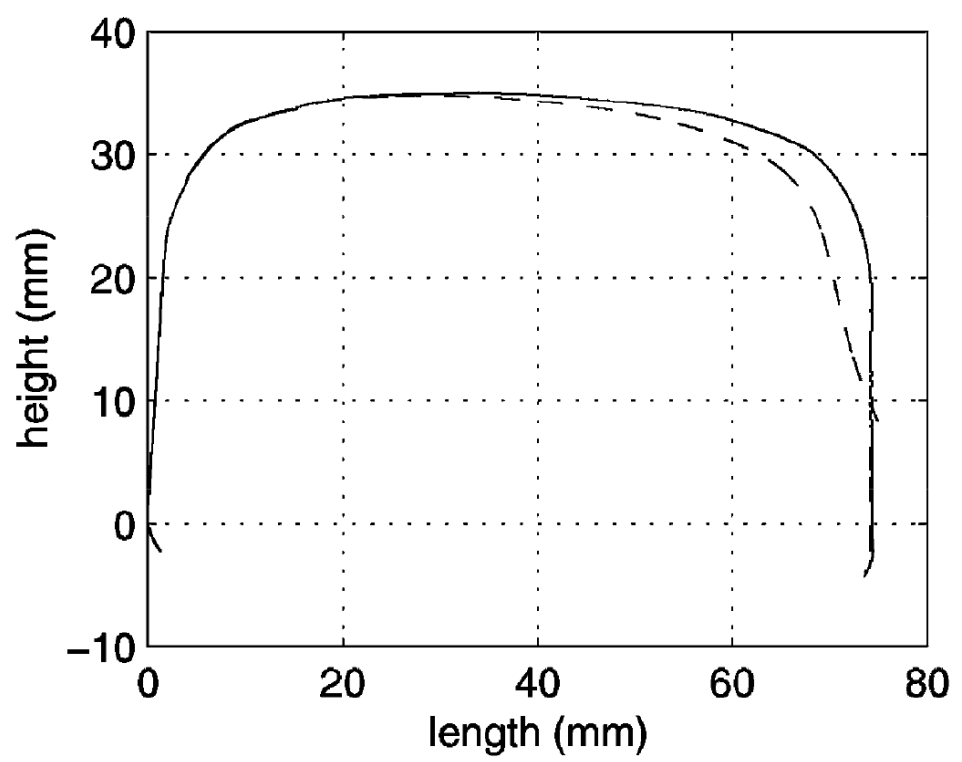

Figure 11: New high rail profiles $(-)$ compared with profiles after 16 months of traffic $(--)$

\section{CONCLUSION}

Traction and wear are two important issues in roll-slip contact, particular in the transportation industry where the driving force of vehicles is obtained by this phenomenon.

The formation of an contact area and pressure distribution is briefly discussed for contacting bodies, with special attention for a sphere-plane contact and wheel-rail contact.

Traction is often considered as a black box, where only the relation between the slip and traction coefficient is known. However this article discusses the formation of traction in the contact area, firstly for static contacts and subsequently for roll-slip contacts.

Wear modeling of roll-slip contacts is based on the theory that the contact area is divided into two regions, a stick and slip region. In the stick area only pure rolling occurs and no wear will arise. In the slip area, the Archard wear model for pure sliding is used. Leading to a wear model for the entire roll-slip contact.

Two types of wear mapping are presented. For the rail industry a wear map based on experimental coupon tests is plotted together with theoretical calculations about the wheel-rail contact. This graph can be used to clarify the location and amount of wear that is observed in field measurements. 


\section{NOMENCLATURE}

$\begin{array}{lll}\mathrm{A} & \text { contact area } & \mathrm{mm}^{2} \\ \mathrm{a} & \text { radius of contact area } & \mathrm{mm} \\ \mathrm{E} & \text { modulus of elasticity } & \mathrm{GPa} \\ \mathrm{E}^{\prime} & \text { effective modulus of elasticity } & \mathrm{GPa} \\ \mathrm{F} & \text { Normal force } & \mathrm{N} \\ \mathrm{H} & \text { Harness } & \mathrm{MPa} \\ \mathrm{K} & \text { wear coefficient } & - \\ \mathrm{Q} & \text { tangential force } & \mathrm{N} \\ \mathrm{R}_{1} & \text { radius sphere } & \mathrm{mm} \\ \mathrm{S} & \text { sliding distance } & \mathrm{mm} \\ \mathrm{S} & \text { slip } & - \\ \mathrm{T} & \text { traction force } & \mathrm{N} \\ \mathrm{u} & \text { lateral shift between wheel and rail } & \mathrm{mm} \\ \mathrm{V} & \text { wear volume } & \mathrm{mm} \\ \mu & \text { coefficient of friction } & - \\ \mathrm{V} & \text { poisson coefficient } & - \\ \sigma & \text { stress } & \mathrm{MPa}\end{array}$

\section{ACKNOWLEDGEMENTS}

The authors would like to acknowledge the financial support of the IWT (Agency for Innovation by Science and Technology $-n^{\circ}$ SB-091510) and the FWO Vlaanderen (Fund for Scientific Research Flanders $-n^{\circ}$ G066908).

\section{REFERENCES}

[1] F.W.Carter, On the action of a locomotive driving wheel. Proc. Roy. Soc.: p. 151-157.1926

[2] A.VanBeek, Advanced engineering design. 2006, Delft.

[3] Segond, D. and A. Tafreshi, Stress analysis of three-dimensional contact problems using the boundary element method. Engineering Analysis with Boundary Elements. 22(3): p. 199-214.1998

[4] Wiest, M., et al., Assessment of methods for calculating contact pressure in wheel-rail/switch contact. Wear. 265(9-10): p. 1439-1445.2008

[5] J.F.Archard, Proc. R. Soc. A 243.1957

[6] Xiao, L., S. Bjorklund, and B.G. Rosen, The influence of surface roughness and the contact pressure distribution on friction in rolling/sliding contacts. Tribology International. 40(4): p. 694-698.2007

[7] Kalker, J.J., Rolling contact phenomena - linear elasticity. CISM International Centre for Mechanical Sciences. Vol. 411. 2001: Springer.

[8] S.Cretu, The influence of roughness on pressure distribution and stress state of concentrated contacts subjected to normal loads. Constructii de masini: p. 1-12.2007

[9] Enblom, R., Deterioration mechanisms in the wheel/rail interface with focus on wear prediction: a literature review. Vehicle System Dynamics: International Journal of Vehicle Mechanics and Mobility. 47(6): p. 661 700.2009

[10] Mindlin, R.D., Compliance of elastic bodies in contact. Journal of Applied Mechanics. 16.1949

[11] K.L.Johnson, Contact Mechanics. 1985, Cambridge Cambridge University Press.

[12] J.F.Archard, Contact and rubbing of flat surfaces. J. Appl. Phys. 24: p. 981-988.1953

[13] Bolton, P.J. and P. Clayton, ROLLING SLIDING WEAR DAMAGE IN RAIL AND TYRE STEELS. Wear. 93(2): p. $145-165.1984$

[14] Olfsson, U. and I. Telliskivi, Wear, plastic deformation and friction of two rail steels-a full-scale test and a laboratory study. Wear. 254(1-2): p. 80-93.2003

[15] Lewis R. and O. U., Mapping rail wear regimes and transitions. Wear. 257(7-8): p. 721-729.2004

[16] Lim, S.C., Recent developments in wear-mechanism maps. Tribology International. 31(1-3): p. 87-97.1998

[17] Danks, D. and P. Clayton, COMPARISON OF THE WEAR PROCESS FOR EUTECTOID RAIL STEELS FIELD AND LABORATORY TESTS. Wear. 120(2): p. 233-250.1987 